\title{
ON THE $x$-COORDINATES OF PELL EQUATIONS WHICH ARE FIBONACCI NUMBERS II
}

\author{
BIR KAFLE, FLORIAN LUCA, AND ALAIN TOGBÉ
}

\begin{abstract}
For an integer $d \geq 2$ which is not a square, we show that there is at most one value of the positive integer $x$ participating in the Pell equation $x^{2}-d y^{2}= \pm 4$ which is a Fibonacci number, except when $d=2,5$, cases in which we have exactly two values of $x$ being members of the Fibonacci sequence.
\end{abstract}

\section{INTRODUCTION}

Let $\left\{F_{m}\right\}_{m \geq 0}$ be the Fibonacci sequence given by $F_{m+2}=F_{m+1}+F_{m}$, for $m \geq 0$, where $F_{0}=0$ and $F_{1}=1$. A few terms of this sequence are

$$
0,1,1,2,3,5,8,13,21,34,55,89,144,233,377,610,987,1597,2584,4181,6765, \ldots
$$

The Fibonacci numbers are well-known for possessing wonderful and amazing properties (see [18, pp. 53-56] and [7] as well as their extensive annotated bibliographies for additional references and history).

Let $d>1$ be a positive integer which is not a perfect square. Consider the Pell equation

$$
x^{2}-d y^{2}= \pm 1 .
$$

All its positive integer solutions $(x, y)$ are given by

$$
x_{n}+y_{n} \sqrt{d}=\left(x_{1}+y_{1} \sqrt{d}\right)^{n},
$$

for some positive integer $n$, where $\left(x_{1}, y_{1}\right)$ is the smallest positive solution. In [12], the second and third author studied the positive integers $n$ such that $x_{n}=F_{m}$ is a member of the Fibonacci sequence and proved that for any $d$, there is at most one such $n$, except when $d=2$ for which there are exactly two such values of $n$.

In this paper, we consider the same problem for the Pell equation

$$
X^{2}-d Y^{2}= \pm 4
$$

Before getting to our main result, let us make some numerical observations. It is known that all positive integer solutions $(X, Y)$ of $(2)$ are given by

$$
\frac{X_{n}+Y_{n} \sqrt{d}}{2}=\left(\frac{X_{1}+Y_{1} \sqrt{d}}{2}\right)^{n}
$$

for some positive integer $n$, where $\left(X_{1}, Y_{1}\right)$ is the smallest positive integer solution.

As a toy example, let us start the study of this question with the small values of $m$, namely $m \leq 3$.

Date: August 18, 2016.

2010 Mathematics Subject Classification. 11A25 11B39, 11J86.

Key words and phrases. Pell equation, Lucas numbers, Linear forms in logarithms. 
- If $m=1,2$, then $X_{n}=F_{m}=1$. Using equation (2), we see that $n=1, d=5$, $Y_{n}=1$.

- If $m=3$, then $X_{n}=F_{m}=2$. Using equation (2), we get that $n=1, d=2$, $Y_{n}=2$ and the the sign on the right-hand side is -.

From now on, we only consider the instance $m \geq 4$.

Hence, we study the Diophantine equation

$$
X_{n} \in\left\{F_{m}\right\}_{m \geq 4} .
$$

Of course, for every integer $x \geq 3$ and every $\varepsilon_{1} \in\{ \pm 4\}$ there is a unique squarefree integer $d \geq 2$ such that

$$
x^{2}-d y^{2}=\varepsilon_{1} .
$$

Namely $d$ is the product of all prime factors of $x^{2}-\varepsilon_{1}$ which appear at odd exponents in its factorization. In particular, taking $x=F_{m}$, we get that any Fibonacci number is the $X$-coordinate of the Pell equation corresponding to one of two specific squarefree integers $d$ (according to the sign of $\varepsilon_{1}$ ). Here, we study the square-free integers $d$ such that the sequence $\left\{X_{n}\right\}_{n \geq 1}$ contains at least two Fibonacci numbers. Our result is the following.

Theorem 1.1. Let $d \geq 2$ be square-free. The Diophantine equation

$$
X_{n} \in\left\{F_{m}\right\}_{m \geq 4}
$$

has at most one solution $(n, m)$ in positive integers. Allowing also $m \in\{1,2,3\}$, the above Diophantine equation still has at most one solution except for $d=2$ and $d=5$, cases in which

$$
n \in\{1,4\}, \quad \text { and } n \in\{1,2\} \text {, }
$$

respectively, are all the solutions of the containment (4).

The organization of this paper is as follows. The proof of Theorem 1.1 proceeds in two cases according to whether $n$ is even or odd. In Section 2, we consider the case in which $n$ even and prove that equation (4) has no other solution in addition to those listed in Theorem 1.1. For this, we transform the main problem into a problem about finding all the rational points on some elliptic curves. This is done by the means of MAGMA. In Section 3, we deal with the case when $n$ is odd. Here, we use Baker's method and the Baker-Davenport reduction method to prove that there is no other solution than those obtained already.

\section{The CASE WHEN $n$ IS EVEN}

Put

$$
\alpha=\frac{X_{1}+Y_{1} \sqrt{d}}{2} \quad \text { and } \quad \beta=\frac{X_{1}-Y_{1} \sqrt{d}}{2} .
$$

One can see that $\alpha \beta=\varepsilon$, so $\beta=\varepsilon \alpha^{-1}$, where $\varepsilon \in\{ \pm 1\}$. With

$$
\alpha^{n}=\frac{X_{n}+Y_{n} \sqrt{d}}{2} \quad \text { and } \quad \beta^{n}=\frac{X_{n}-Y_{n} \sqrt{d}}{2},
$$

we obtain

$$
X_{n}=\alpha^{n}+\beta^{n} .
$$


Thus,

$$
\begin{aligned}
X_{2 n} & =\alpha^{2 n}+\beta^{2 n}=\left(\frac{X_{n}+\sqrt{d} Y_{n}}{2}\right)^{2}+\left(\frac{X_{n}-\sqrt{d} Y_{n}}{2}\right)^{2} \\
& =\frac{1}{2}\left(X_{n}^{2}+d Y_{n}^{2}\right)=\frac{1}{2}\left(X_{n}^{2}+\left(X_{n}^{2}-4 \varepsilon\right)\right)=X_{n}^{2}-2 \varepsilon .
\end{aligned}
$$

Therefore, it suffices to solve the equation

$$
u^{2} \pm 2=F_{m}, \quad \text { where } \quad m \geq 1 .
$$

There are many papers in the literature solving Diophantine equations of the form $F_{n}=f(u)$, for some quadratic polynomial $f(x) \in \mathbb{Q}[x]$ by elementary means. We give only a couple of examples. The only squares in the Fibonacci sequence are $0=F_{0}, 1=F_{1}=F_{2}, 144=F_{12}$. This is a consequence of the work of Ljunggren [8], [10] (see the Introduction to [11]) and was rediscovered by Cohn [2] and Wyler [19]. All triangular numbers in the Fibonacci sequence are $1=F_{1}=F_{2}, 3=$ $F_{4}, 21=F_{8}, 55=F_{10}$ were found by an elementary method by Luo Ming [16]. It is therefore likely that one can find all solutions of equation (5) by elementary means using only congruences and Jacobi symbol manipulations. We preferred a more computational approach using MAGMA, which we now describe. Since the formula

$$
V^{2}-5 U^{2}= \pm 4
$$

holds with $(V, U)=\left(L_{m}, F_{m}\right)$, where $\left\{L_{n}\right\}_{n \geq 0}$ is the Lucas companion of the Fibonacci sequence given by $L_{0}=2, L_{1}=1$ and $L_{n+2}=L_{n+1}+L_{n}$ for all $n \geq 0$, it follows that by replacing $F_{m}$ with $u^{2} \pm 2$ and setting $v=L_{m}$, we obtain

$$
v^{2}=5\left(u^{2} \pm 2\right)^{2} \pm 4 \text {. }
$$

In the right-hand sides of (7) above we have one of four polynomials each of degree 4. Se we are lead to integer points $(u, v)$ on the following four elliptic curves:

$$
\begin{aligned}
& v^{2}=5 u^{4}+20 u^{2}+24 ; \\
& v^{2}=5 u^{4}-20 u^{2}+24 ; \\
& v^{2}=5 u^{4}+20 u^{2}+16 ; \\
& v^{2}=5 u^{4}-20 u^{2}+16 .
\end{aligned}
$$

We used MAGMA to determine the integer points $(u, v)$ on these elliptic curves. We obtained:

$$
\begin{aligned}
& ( \pm 1, \pm 7), \text { for curve }(8) \\
& ( \pm 1, \pm 3), \text { for curve }(9) \\
& (0, \pm 4), \text { for curve }(10)
\end{aligned}
$$

$$
(0, \pm 4),( \pm 1, \pm 1),( \pm 2, \pm 4),( \pm 6, \pm 76), \text { for curve }(11) .
$$

As $F_{m}=u^{2} \pm 2$, we get that $X=X_{n}=F_{m} \in\{2,3,34\}$. Using the equation $X^{2}-d Y^{2}= \pm 4$, we see that:

- for $X=2$, we get $(Y, d, \varepsilon, n)=(2,2,-1,1)$;

- for $X=3$, we get $(Y, d, \varepsilon, n)=(1,5,1,2),(1,13,-1,1)$; 
- for $X=34$, we get $(Y, d, \varepsilon, n)=(24,2,1,4),(2,290,-1,1)$.

Since $n$ is even, the only acceptable cases are $(n, d)=(2,5),(4,2)$. In both cases,

$$
X_{1}^{2}-d Y_{1}^{2}=-4 \text {. }
$$

So far, we have seen that if $X_{n} \in\left\{F_{m}\right\}_{m \geq 1}$ holds for some even $n$, then we must have $(n, d)=(2,5),(4,2)$. Since we are searching for solutions to the problem when $X_{n} \in\left\{F_{m}\right\}_{m \geq 1}$ holds for at least two values of $n$, it follows that in each of the above two cases, the other value of $n$ must be odd. This leads to

$$
X_{n}^{2}-d Y_{n}^{2}=-4 \quad \text { with } \quad d \in\{2,5\} .
$$

When $d=5$, it is well-known that $\left(X_{n}, Y_{n}\right)=\left(L_{n}, F_{n}\right)$, and furthermore, $n$ must be odd. Hence, we get $L_{n}=F_{m}$, whose only convenient solution is $n=1$. For $d=2$, we rewrite our equation as

$$
2 Y_{n}^{2}=X_{n}^{2}+4=F_{m}^{2}+4
$$

Multiplying the above relation with $L_{m}^{2}=5 F_{m}^{2} \pm 4$, we get

$$
\left(2 Y_{n} L_{m}\right)^{2}=2\left(F_{m}^{2}+4\right)\left(5 F_{m}^{2} \pm 4\right) .
$$

Setting $u:=F_{m}$ and $v:=2 Y_{n} L_{m}$, we are led to solving the equations

$$
v^{2}=2\left(u^{2}+4\right)\left(5 u^{2}+4\right)=10 u^{4}+48 u^{2}+32
$$

and

$$
v^{2}=2\left(u^{2}+4\right)\left(5 u^{2}-4\right)=10 u^{4}+32 u^{2}-32
$$

in positive integers $(u, v)$. Only equation (13) gives us the solution $(u, v)=(2,16)$ which leads to $X_{n}=F_{m}=2=F_{3}$, and $Y_{n}=2$.

Lemma 2.1. Assume that $X^{2}-d Y^{2}= \pm 4$ and that $X_{n}=F_{m}$ for some even $n$. Then, $(n, d)=(2,5),(4,2)$. Additionally, if $d=2$ and $d=5$, the only solutions of $X_{n}=F_{m}$ (regardless of the parity of $n$ ) are $n=1,4$, and $n=1,2$, respectively.

\section{THE CASE $n$ ODD}

3.1. Preliminary considerations. From now on, $d>2$ and $d \neq 5$. Now suppose that $n_{1}<n_{2}$ are odd integers such that $X_{n_{1}}=F_{m_{1}}$ and $X_{n_{2}}=F_{m_{2}}$ for some positive integers $m_{1}<m_{2}$. Since $n_{1}$ and $n_{2}$ are odd, we have

$$
\operatorname{gcd}\left(X_{n_{1}}, X_{n_{2}}\right)=X_{\operatorname{gcd}\left(n_{1}, n_{2}\right)} .
$$

Further, $\operatorname{gcd}\left(F_{m_{1}}, F_{m_{2}}\right)=F_{\operatorname{gcd}\left(m_{1}, m_{2}\right)}$. Thus, by replacing $n_{1}$ with $\operatorname{gcd}\left(n_{1}, n_{2}\right)$ and $m_{1}$ by $\operatorname{gcd}\left(m_{1}, m_{2}\right)$, we may assume that $n_{1} \mid n_{2}$ and $m_{1} \mid m_{2}$. Thus, we put $n_{2}=n_{1} n$ and $m_{2}=m_{1} t$ for some positive integers $n>1, t>1$. Clearly, $n$ is odd. Further, we replace $\left(X_{n_{1}}, Y_{n_{1}}\right)$ by $\left(X_{1}, Y_{1}\right)$, therefore we replace $\left(\alpha^{n_{1}}, \beta^{n_{1}}\right)$ by $(\alpha, \beta)$. We obtain

$$
X_{1}=\alpha+\beta=F_{m_{1}}
$$

and

$$
X_{n}=\alpha^{n}+\beta^{n}=F_{m_{1} t} .
$$


Since $n_{1}$ is odd, it follows that $\varepsilon^{n_{1}}=(\alpha \beta)^{n_{1}}=\varepsilon$ is preserved under the above replacements. We put $(\gamma, \delta)=((1+\sqrt{5}) / 2,(1-\sqrt{5}) / 2)$ for the golden section and its conjugate. The formula

$$
F_{k}=\frac{\gamma^{k}-\delta^{k}}{\sqrt{5}} \quad \text { holds for all } \quad k \geq 1
$$

With these notations, the following inequalities hold.

Lemma 3.1. We have the following estimates:

$$
\begin{gathered}
\left|\alpha-\frac{1}{\sqrt{5}} \gamma^{m_{1}}\right|<\frac{6}{\gamma^{m_{1}}}, \\
\gamma^{m_{1} t-2}<\alpha^{n}<\gamma^{m_{1} t}, \\
\left|\sqrt{5} \gamma^{-m_{1} t} \alpha^{n}-1\right|<\frac{10}{\gamma^{2 m_{1} t}} .
\end{gathered}
$$

Proof. Using the equation (14) and the Binet formula (16) for the Fibonacci numbers, we obtain

$$
\alpha+\beta=\frac{\gamma^{m_{1}}-\delta^{m_{1}}}{\sqrt{5}}
$$

We deduce that

$$
\alpha=\frac{1}{\sqrt{5}} \gamma^{m_{1}}-\beta-\frac{1}{\sqrt{5}} \delta^{m_{1}}
$$

Since $\alpha>3$ (because $d>2$ ) and $|\beta|<1$, we have

$$
\frac{2 \alpha}{3}<\alpha+\beta<2 \alpha \text {. }
$$

Further,

$$
\gamma^{m_{1}-2}<F_{m_{1}}<\gamma^{m_{1}-1}
$$

inequality which can be deduced easily from the Binet formula (16). Thus, from (14), we deduce

$$
\frac{2 \alpha}{3}<F_{m_{1}}<\gamma^{m_{1}-1} \quad \text { so } \quad \alpha<\frac{3}{2} \gamma^{m_{1}-1}<\gamma^{m_{1}}
$$

as well as

$$
\gamma^{m_{1}-2}<F_{m_{1}}<2 \alpha \text { and so } \frac{1}{2} \gamma^{m_{1}-2}<\alpha .
$$

This leads to

$$
\frac{1}{2} \gamma^{m_{1}-2}<\alpha<\gamma^{m_{1}}
$$

So, we get

$$
\gamma^{m_{1}-4}<\alpha<\gamma^{m_{1}}
$$

Therefore, we obtain from (21), that

$$
\left|\alpha-\frac{1}{\sqrt{5}} \gamma^{m_{1}}\right|=\left| \pm \frac{1}{\alpha}+\frac{1}{\sqrt{5}}( \pm \gamma)^{-m_{1}}\right| \leq \frac{1}{\gamma^{m_{1}}}\left(\frac{1}{\sqrt{5}}+2 \gamma^{2}\right)<\frac{6}{\gamma^{m_{1}}},
$$

which proves (17). On the other hand, we use equation (15) to get

$$
\alpha^{n}=\frac{1}{\sqrt{5}} \gamma^{m_{1} t}-\beta^{n}-\frac{1}{\sqrt{5}} \delta^{m_{1} t} .
$$


Similarly as above, we have

$$
\gamma^{m_{1} t-2}<F_{m_{1} t}=\alpha^{n}+\beta^{n}<\gamma\left(\alpha^{n}+\beta^{n}\right)=\gamma F_{m_{1} t}<\gamma^{m_{1} t} .
$$

Thus, one can see that

$$
\gamma^{m_{1} t-2}<\alpha^{n}<\gamma^{m_{1} t}
$$

which is (18). Estimate (18) together with (23) leads to

$$
\left|\alpha^{n}-\frac{1}{\sqrt{5}} \gamma^{m_{1} t}\right|=\left| \pm \frac{1}{\alpha^{n}}+\frac{1}{\sqrt{5}}( \pm \gamma)^{m_{1} t}\right| \leq \frac{1}{\gamma^{m_{1} t}}\left(\frac{1}{\sqrt{5}}+\gamma^{2}\right)<\frac{2 \sqrt{5}}{\gamma^{m_{1} t}} ;
$$

which gives us

$$
\left|\sqrt{5} \gamma^{-m_{1} t} \alpha^{n}-1\right|<\frac{10}{\gamma^{2 m_{1} t}}
$$

This completes the proof of Lemma 3.1.

3.2. An inequality among $n$ and $t$. In this subsection, we prove the following result that helps to compare $n$ and $t$.

Lemma 3.2. We have $n>t$.

Proof. We have that

$$
(\alpha, \beta)=\left(\frac{F_{m_{1}}+\sqrt{F_{m_{1}}^{2}-\varepsilon_{1}}}{2}, \frac{F_{m_{1}}-\sqrt{F_{m_{1}}^{2}-\varepsilon_{1}}}{2}\right),
$$

where $\varepsilon_{1}=4 \varepsilon$. By induction on $n$, one can readily prove that the two sequences $\left\{X_{n}\right\}_{n \geq 1}$ and $\left\{F_{m_{1} n}\right\}_{n \geq 1}$ satisfy

$$
\begin{aligned}
X_{n} & =F_{m_{1}} X_{n-1}+(-\varepsilon) X_{n-2} \\
F_{m_{1} n} & =L_{m_{1}} F_{m_{1}(n-1)}+(-1)^{m_{1}-1} F_{m_{1}(n-2)} .
\end{aligned}
$$

for all $n \geq 3$. Further, we have

$$
X_{1}=F_{m_{1}}, \quad X_{2}=F_{m_{1}}^{2} \pm 2 \leq F_{m_{1}}^{2}+2<F_{2 m_{1}} .
$$

The last inequality in (28) follows because $F_{2 m_{1}}=F_{m_{1}} L_{m_{1}}$ and $L_{m_{1}}>2 F_{m_{1}}$, for all $m_{1} \geq 4$, inequality which is obvious because of the formula $L_{m_{1}}=2 F_{m_{1}}+F_{m_{1}-3}$, which can be proved by induction on $m_{1} \geq 4$. We now prove by induction on $n$ that the inequality

$$
X_{n}<F_{m_{1} n} \quad \text { holds for all } \quad n \geq 2 .
$$

This together with (15) will give us the desired conclusion that $t<n$.

The inequality $X_{n}<F_{m_{1} n}$ holds with $n=2$ by (28) and we also have $X_{1}=F_{m_{1}}$ (so when $n=1$ we have equality). Suppose that $n \geq 3$. Since $L_{m_{1}}>2 F_{m_{1}}$, for all $m_{1} \geq 4$, the desired inequality follows by induction on $n$ from the two recurrences (26) and (27) when $m_{1}$ is odd. When $m_{1}$ is even, again by induction on $n$, we have

$$
\begin{aligned}
F_{m_{1} n} & =L_{m_{1}} F_{m_{1}(n-1)}-F_{m_{1}(n-2)} \\
& =\left(L_{m_{1}}-1\right) F_{m_{1}(n-1)}+\left(F_{m_{1}(n-1)}-F_{m_{1}(n-2)}\right) \\
& \geq F_{m_{1}} F_{m_{1}(n-1)}+F_{m_{1}(n-2)}>F_{m_{1}} X_{n-1}+X_{n-2}=X_{n},
\end{aligned}
$$

which is what we wanted to prove. 
3.3. An inequality among $m_{1}$ and $n$. The following result will help to compare $m_{1}$ and $n$.

Lemma 3.3. We have $\gamma^{m_{1}}<6 n^{2}$.

Proof. We shall show that

$$
F_{m_{1}} \mid n^{2} \pm t^{2}
$$

The right-hand side above is nonzero by Lemma 3.2. Divisibility (29) will immediately imply the desired conclusion since then $\gamma^{m_{1}-2}<F_{m_{1}} \leq n^{2} \pm t^{2}<2 n^{2}$ by Lemma 3.2, so $\gamma^{m_{1}}<2 \gamma^{2} n^{2}<6 n^{2}$, which is what we want.

Recall that the Dickson polynomial

$$
D_{n}(x, v)=\sum_{p=0}^{\lfloor n / 2\rfloor} \frac{n}{n-p}\left(\begin{array}{c}
n-p \\
p
\end{array}\right)(-v)^{p} X^{n-2 p}
$$

satisfies

$$
D_{n}(u+v / u, v)=u^{n}+(v / u)^{n} .
$$

Taking $n$ to be odd, $u=\alpha, v=\varepsilon$, we get that

$$
\frac{X_{n}}{X_{1}}=\frac{\alpha^{n}+\beta^{n}}{\alpha+\beta}=\frac{D_{n}\left(X_{1}, \varepsilon\right)}{X_{1}} \equiv(-\varepsilon)^{\lfloor n / 2\rfloor} n \quad\left(\bmod X_{1}\right),
$$

by (30). Since $X_{1}=F_{m_{1}}$ and $X_{n}=F_{m_{1} t}$, we get that

$$
\frac{F_{m_{1} t}}{F_{m_{1}}} \equiv \pm n \quad\left(\bmod F_{m_{1}}\right) .
$$

When $t$ is odd, the left-hand above is congruent to $\pm t$ modulo $F_{m_{1}}$, a fact which can be proved invoking again properties of the Dickson polynomials. But we prefer a direct approach. Given two algebraic integers $\eta, \zeta$ and an integer $m$ we say that $\eta \equiv \zeta(\bmod m)$ if $(\eta-\zeta) / m$ is an algebraic integer. Then, $\gamma^{m_{1}} \equiv \delta^{m_{1}}\left(\bmod F_{m_{1}}\right)$, therefore

$$
\frac{F_{m_{1} t}}{F_{m_{1}}}=\frac{\gamma^{m_{1} t}-\delta^{m_{1} t}}{\gamma^{m_{1}}-\delta^{m_{1}}}=\gamma^{m_{1}(t-1)}+\cdots+\delta^{m_{1}(t-1)} \equiv t \gamma^{m_{1}(t-1)} \quad\left(\bmod F_{m_{1}}\right) .
$$

The same congruence holds if we replace $\gamma$ by $\delta$ and multiplying them we get

$$
\left(\frac{F_{m_{1} t}}{F_{m_{1}}}\right)^{2} \equiv t^{2}(\gamma \delta)^{m_{1}(t-1)} \equiv \pm t^{2} \quad\left(\bmod F_{m_{1}}\right) .
$$

By $(31)$, the left-hand side above is congruent to $n^{2}\left(\bmod F_{m_{1}}\right)$, which together with (32) leads to divisibility relation (29), which is what we wanted.

3.4. Bounding $n$ and $m_{1}$. The next result will give us upper bounds for $n$ and $m_{1}$. But before this, we recall the following result due to Matveev [14]. Let $\mathbb{L}$ be an algebraic number field and $d_{\mathbb{L}}$ be the degree of the field $\mathbb{L}$. Let $\eta_{1}, \eta_{2}, \ldots, \eta_{l} \in \mathbb{L}$ not 0 or 1 and $d_{1}, \ldots, d_{l}$ be nonzero integers. We put

$$
D=\max \left\{\left|d_{1}\right|, \ldots,\left|d_{l}\right|, 3\right\}
$$

and put

$$
\Lambda=\prod_{i=1}^{l} \eta_{i}^{d_{i}}-1
$$


Let $A_{1}, \ldots, A_{l}$ be positive integers such that

$$
A_{j} \geq h^{\prime}\left(\eta_{j}\right):=\max \left\{d_{\mathbb{L}} h\left(\eta_{j}\right),\left|\log \eta_{j}\right|, 0.16\right\}, \quad \text { for } \quad j=1, \ldots l,
$$

where for an algebraic number $\eta$ we write $h(\eta)$ for its Weil height.

Theorem 3.1. If $\Lambda \neq 0$ and $\mathbb{L} \subset \mathbb{R}$, then

$$
\log |\Lambda|>-1.4 \cdot 30^{l+3} l^{4.5} d_{\mathbb{L}}^{2}\left(1+\log d_{\mathbb{L}}\right)(1+\log D) A_{1} A_{2} \cdots A_{l} .
$$

We now use the above result to prove the following lemma.

Lemma 3.4. We have $n<2.9 \times 10^{15}$. Additionally, we have $m_{1} \leq 154$.

Proof. We take

$$
\Lambda:=\sqrt{5} \gamma^{-m_{1} t} \alpha^{n}-1
$$

This is nonzero, since if it were, then $\sqrt{5}=\gamma^{m_{1} t} \alpha^{-n}$ would be a unit, which is false since it belongs to $\mathbb{L}=\mathbb{Q}(\sqrt{5}, \alpha)$ and its norm from $\mathbb{L}$ to $\mathbb{Q}$ is $5^{2}$. We use Theorem 3.1 to get a lower bound for $|\Lambda|$. We take $l=3$,

$$
\eta_{1}=\sqrt{5}, \quad \eta_{2}=\gamma, \quad \eta_{3}=\alpha, \quad d_{1}=1, \quad d_{2}=-m_{1} t, \quad d_{3}=n .
$$

Clearly, $d_{\mathbb{L}} \in\{2,4\}$. We have $h\left(\eta_{1}\right)=\log 5, h\left(\eta_{2}\right)=(\log \gamma) / 2, h(\alpha)=(\log \alpha) / 2$. Thus, we can take $A_{1}=2 \log 5, A_{2}=2 \log \gamma, A_{3}=2 \log \alpha$. Since $d \geq 3$ and $d \neq 5$, we have that $\alpha>\gamma^{2}$, so inequality (18) gives that

$$
\gamma^{2 n}<\alpha^{n}<\gamma^{m_{1} t}
$$

so $n<m_{1} t$. Hence, we can take $D:=m_{1} t$. Theorem 3.1 gives now that

(33) $-\log |\Lambda|<2.8 \times 30^{6} \times 3^{4.5} \times 4^{2}(1+\log 4)(2 \log 5)(2 \log \gamma)(2 \log \alpha)\left(1+\log \left(m_{1} t\right)\right)$.

On the other hand, inequalities (18) and (19) give

$$
|\Lambda|<\frac{10}{\gamma^{2 m_{1} t}}<\frac{10 \gamma^{4}}{\alpha^{2 n}}<\frac{80}{\alpha^{2 n}} \quad \text { so } \quad-\log |\Lambda|>2 n \log \alpha-\log 80 .
$$

Putting (33) and (34) together, we get

$$
n<2.8 \times 30^{6} \times 3^{4.5} \times 4^{2}(1+\log 4)(2 \log 5)(2 \log \gamma)\left(1+\log \left(m_{1} t\right)\right)+\frac{\log 80}{\log \alpha} .
$$

Since $\alpha>2+\sqrt{3}, t<n$ (by Lemma 3.2) and $m_{1}<\log \left(6 n^{2}\right) / \log \gamma$ (by Lemma $3.3)$, we get

$$
n<3.4 \times 10^{13}\left(1+\log \left(n \log \left(6 n^{2}\right) / \log \gamma\right)\right),
$$

giving $n<2.9 \times 10^{15}$. Additionally, $F_{m_{1}}<2 n^{2}<10^{32}$, so $m_{1} \leq 154$.

3.5. The final step. For each $m_{1} \in[4,154]$ and $\varepsilon \in\{ \pm 1\}$, we calculate

$$
\alpha=\frac{F_{m_{1}}+\sqrt{F_{m_{1}}^{2}-4 \varepsilon}}{2} .
$$

We put

$$
\Gamma:=n \log \alpha-m_{1} t \log \gamma+\log (\sqrt{5}) .
$$

Note that $e^{\Gamma}-1=\Lambda$. Since $t \geq 2, m_{1} \geq 4$, we have that $m_{1} t \geq 8$, so by (19), we have that

$$
|\Lambda|<\frac{10}{\gamma^{2 m_{1} t}}<\frac{1}{2} .
$$


By a classical inequality, this leads to

$$
|\Gamma| \leq 2|\Lambda| \leq \frac{20}{\gamma^{2 m_{1} t}}
$$

Inequality (35) is suitable to apply the reduction algorithm. Note that

$$
n<m_{1} t<m_{1} n<4.5 \times 10^{17}:=M .
$$

So in order to deal with the remaining cases, for $m_{1} \in[4,154]$, we used a Diophantine approximation algorithm called the Baker-Davenport reduction method. The following lemma is a slight modification of the original version of Baker-Davenport reduction method. (See [6, Lemma 5a]).

Lemma 3.5. Assume that $M$ is a positive integer. Let $P / Q$ be the convergent of the continued fraction expansion of $\kappa$ such that $Q>6 M$ and let

$$
\eta=\|\mu Q\|-M \cdot\|\kappa Q\|,
$$

where $\|\cdot\|$ denotes the distance from the nearest integer. If $\eta>0$, then there is no solution of the inequality

$$
0<m \kappa-n+\mu<A B^{-m}
$$

in integers $m$ and $n$ with

$$
\frac{\log (A Q / \eta)}{\log B} \leq m \leq M
$$

As

$$
0<n \log \alpha-m_{1} t \log \gamma+\log (\sqrt{5})<\frac{20}{\gamma^{2 n}},
$$

we apply Lemma 3.5 with

$$
\kappa=\frac{\log \alpha}{\log \gamma}, \quad \mu=\frac{\log (\sqrt{5})}{\log \gamma}, \quad A=\frac{20}{\log \gamma}, \quad B=\gamma^{2}, \quad M=4.5 \cdot 10^{17} .
$$

The program was developed in PARI/GP running with 200 digits. For the computations, if the first convergent such that $q>6 M$ does not satisfy the condition $\eta>0$, then we use the next convergent until we find the one that satisfies the conditions. In one minute, all the computations were done. In all cases, we obtained $m_{1} t \leq 157$. We set $M=157$ and the second run of the reduction method yields no improvement.

For each $t$, we choose $n$ odd such that inequalities (18) holds (if it exists) and with this $n$, we check whether the equality

$$
X_{n}=D_{n}\left(F_{m_{1}}, \varepsilon\right)=F_{m_{1} t},
$$

holds where the polynomial $D(x, v)$ is shown at (30). If it does, we have found another solution to our original problem. We wrote a program in Maple that we ran through the remaining range and found no new solutions.

\section{ACKnowledgements}

The authors are grateful to the anonymous referee for the careful reading the manuscript. The first and the third authors are partially supported by Purdue University Northwest. 


\section{REFERENCES}

[1] Y. Bugeaud, M. Mignotte, S. Siksek, Classical and modular approaches to exponential Diophantine equations I. Fibonacci and Lucas perfect powers, Ann. of Math. 163 (2006), 969-1018.

[2] J. H. E. Cohn, On square Fibonacci numbers, J. London Math. Soc. 39 (1964), 537-540.

[3] J. H. E. Cohn, The Diophantine equation $x^{4}-D y^{2}=1$, Quart. J. Math. Oxford (2) 26 (1975), 279-281.

[4] J. H. E. Cohn, The Diophantine equation $x^{4}-D y^{2}=1$, II, Acta Arithmetica 78 (1997), 401-403.

[5] R. D. Carmichael, On the numerical factors of the arithmetic forms $\alpha^{n} \pm \beta^{n}$, Ann. Math. (2) 15 (1913), 30-70.

[6] A. Dujella, A. Pethö, A generalization of a theorem of Baker and Davenport, Quart. J. Math. Oxford Ser. (2) 49 (1998), 291-306.

[7] D. Kalman, R. Mena, The Fibonacci numbers-exposed, Math. Magazine 76 (3) (2003), $167-181$.

[8] W. Ljunggren, Über die unbestimmte Gleichung $A x^{2}-B y^{4}=C$, Arch. f. Mat. og Naturvid. 41 (1938), No. 10, 18 pp.

[9] W. Ljunggren, Über die Gleichung $x^{4}-D y^{2}=1$, Arch. Math. Naturv. 45 (1942), 61-70.

[10] W. Ljunggren, On the diophantine equation $x^{2}+4=A y^{2}$, Norske Vid. Selsk. Forh., Trondheim. 24 (1951), 82-84.

[11] W. Ljunggren, Collected Papers of Wilhelm Ljunggren, Vol . 1, 2 (Paulo Ribenboim, ed.), Queens Papers in Pure and Applied Math. 115, Queens University, Kingston, ON, 2003.

[12] F. Luca, A. Togbé, On the $x$-coordinates of Pell equations which are Fibonacci numbers. To appear in Mathematica Scandinavica.

[13] D. Marques, A. Togbé, On terms of linear recurrence sequences with only one distinct block of digits, Colloquium Mathematicum 124 (2011), 145-155.

[14] E. M. Matveev, An explicit lower bound for a homogeneous rational linear form in logarithms of algebraic numbers, II, Izv. Ross. Akad. Nauk Ser. Mat. 64 (2000), 125-180. English translation in Izv. Math. 64 (2000), 1217-1269.

[15] W. McDaniel, The GCD in Lucas sequences and Lehmer number sequences, Fibonacci Quarterly 29 (1991), 24-29.

[16] L. Ming, On triangular Fibonacci numbers, Fibonacci Quarterly 27 (1989), 98-108.

[17] R. Obláth, Une propriété des puissances parfaites, Mathesis 65 (1956), 356-364.

[18] A. S. Posamentier, I. Lehmann, The Fabulous Fibonacci Numbers, Prometheus Books, New York, 2007.

[19] O. Wyler and A. P. Rollett, Advanced problems and solutions: Solution: 5080, Amer. Math. Monthly 71 (1964), 220-222.

[20] K. Yu: p-adic logarithmic forms and group varieties II, Acta Arith. 89 (1999), 337-378.

Mathematics Department, Purdue University Northwest, 1401 S, U.S. 421, Westville IN 46391 USA

E-mail address: bkafle@pnw.edu

School of Mathematics, University of the Witwatersrand, Private Bag X3, Wits 2050, South Africa and Max Planck Institute for Mathematics Vivatgasse 7, 53111 Bonn, GERMAny

E-mail address: florian.luca@wits.ac.za

Mathematics Department, Purdue University Northwest, 1401 S, U.S. 421, Westville IN 46391 USA

E-mail address: atogbe@pnw.edu 